\title{
Development of noninvasive biomarkers of response to proteasome inhibitor therapy (ixazomib) by imaging disrupted protein homeostasis in mouse models of solid tumors
}

Yanan Zhu ${ }^{1}$, Rajiv Ramasawmy ${ }^{1}$, Sean Peter Johnson ${ }^{1}$, Valerie Taylor ${ }^{1}$, Alasdair Gibb ${ }^{6}$, R Barbara Pedley $^{3}$, Nibedita Chattopadhyay ${ }^{2}$, Mark F Lythgoe ${ }^{1}$, Xavier Golay ${ }^{4}$, Daniel Bradley ${ }^{5}$, Simon Walker-Samuel ${ }^{1}$

${ }^{1} \mathrm{UCL}$ Centre for Advanced Biomedical Imaging, Division of Medicine, University College London, ${ }^{2}$ Cancer Pharmacology, Takeda Pharmaceutical International Corporation, ${ }^{3} \mathrm{UCL}$ Cancer Institute, University College London, ${ }^{4}$ Institute of Neurology, University College London, ${ }^{5}$ The Biomedical Imaging Group, Takeda Pharmaceutical International Corporation, ${ }^{6}$ Neuroscience, Physiology \& Pharmacology, University College London

\section{Running title}

Imaging biomarkers of protein homeostasis

\section{Corresponding author}

Simon Walker-Samuel

Centre for Advanced Biomedical Imaging, University College London

Lower Ground Floor, Paul O'Gorman Building, 72 Huntley Street

WC1E 6DD London

Tel.: $\quad$ +44 2076796329

Email: simon.walkersamuel@ucl.ac.uk

Key words: protein, homeostasis, ixazomib, Ninlaro, colorectal cancer, CEST MRI, imaging biomarkers

\section{Declaration of the conflicts of interest:}

The project was partly funded by Takeda Pharmaceuticals Company Limited. 


\begin{abstract}
With clinically-approved proteasome inhibitors now a standard of care for multiple myeloma, and increasing interest in their use in solid tumors, methods for monitoring therapeutic response in vivo are critically required. Here, we show that tumor protein homeostasis can be noninvasively monitored, using chemical exchange (CEST) magnetic resonance imaging (MRI) as a surrogate marker for proteasome inhibition, alongside diffusion MRI and relaxometry. We show that the in vivo CEST signal associated with amides and amines increases in proportion to proteasome inhibitor dose (ixazomib) and the magnitude of therapeutic effect in colorectal cancer xenografts. Moreover, we show that SW1222 and LS174T human colorectal cancer cell lines demonstrate differing sensitivities to ixazomib, which was reflected in our MRI measurements. We also found evidence of a mild stimulation in tumor growth at low ixazomib doses. Our results therefore identify CEST MRI as a promising method for safely and noninvasively monitoring changes in tumor protein homeostasis.
\end{abstract}




\section{Introduction}

Loss of protein homeostasis is associated with a range of pathological conditions including most forms of dementia (1), amyloidosis (2) and cancers (3). In cancer, malignant cells exhibit an abnormally high turnover of protein (3), which is most notably exploited as a therapeutic target by proteasome inhibitors (PIs) (4). By inhibiting the action of proteasomes, PIs disrupt protein homeostasis, resulting in apoptotic cell death. PIs are now in routine clinical use for treating haematological malignancies (5-7).

Ixazomib (Ninlaro/MLN2238/MLN9708, Takeda Pharmaceuticals Company Limited) is the first orally-available PI therapy $(8,9)$ with approval for use in the United States, Europe and Japan for combinational treatment in multiple myeloma $(9,10)$. Ixazomib has also shown promise in solid tumor rodent models, including colorectal tumors, and there is an increasing research interest in this area $(8,11-13)$.

Here, we propose a new set of noninvasive imaging biomarkers for detecting disrupted protein homeostasis, resulting from PI therapy, based on magnetic resonance imaging (MRI). In particular, we have focused on chemical exchange saturation transfer (CEST) MRI, which has previously been used to detect changes in protein content in cancer (14-16). CEST image contrast can be tuned to reflect the exchange of protons between water and various chemical groups, including amides (e.g. in protein backbones) (17), amines and hydroxyls. This enables the small signal from solutes containing such groups to be amplified via the much larger water pool signal. We hypothesized that successful proteasome inhibition, and associated protein accumulation $(4,18)$, would result in an increase in CEST signal associated with amide and amine groups.

Alongside CEST, we evaluated a range of quantitative, noninvasive MRI measurements (diffusion MRI, $T_{1}$ and $T_{2}$ relaxometry), in LS174T and SW1222 xenograft models of human colorectal carcinoma. In vivo measurements of acute (up to 72 hours) dosing with ixazomib, at three different dose concentrations, were undertaken and compared with gold-standard histological measures (19-21). Biological validation studies, such as undertaken here, are a vital component of the translation pathway for imaging biomarkers into the clinic (22).

The aims of our study were to: 1 ) determine the efficacy of ixazomib in colorectal carcinoma cell lines and murine xenografts; and 2) evaluate the ability of noninvasive MRI measurements to evaluate this response in vivo, with a view to their translation into the clinic.

\section{Materials and Methods}

Details of cell culturing methods for SW1222 and LS174T cells are provided in the supplemental methods.

\section{In vitro MTT assay}

MTT (Vybrant MTT Cell Proliferation Assay Kit, Life Technologies) reagent at $12 \mathrm{mM}$ was prepared with a dilution of $1 \mathrm{~mL}$ of sterile PBS to one $5 \mathrm{mg}$ MTT powder, according to manufacturer's instructions. Before treatment, 10,000 in $100 \mu \mathrm{L}$ of complete media per well were seeded, for 9 hours, in a clear polystyrene 96 -well flat bottom microplate (Greiner Bio-One).

Ixazomib was diluted from $10 \mathrm{mM}$ stock solution in complete media from 400 to $1.6 \mathrm{nM}$ using 2-fold serial dilution. Media in each well was removed and replaced with $90 \mu \mathrm{L}$ of ixazomib, with corresponding concentration. Cells were left for 24, 48 or 72 hours, then labeled with $10 \mu \mathrm{L}$ of the $12 \mathrm{mM}$ MTT reagent, and incubated for 2 hours at $37^{\circ} \mathrm{C}$. After the incubation period, the media was removed and replaced with $100 \mu \mathrm{L}$ of DMSO per well, and mixed thoroughly using an orbital plate shaker for 2 minutes. The microplate was incubated for $37^{\circ} \mathrm{C}$ for 10 min and were read using a microplate absorbance reader at $540 \mathrm{~nm}$. 
Cell viability was estimated from the absorbance measure (unitless), a measure of cell viability as a percentage of the mean absorbance of control cells. These data were fitted to the modified Hill equation, from which the $E C_{50}$ (half-maximal stimulatory concentration) and $I C_{50}$ (half-maximal inhibitory concentration) were estimated (see supplemental methods for details).

\section{Animal Models}

All in vivo experiments were performed in accordance with the UK Home Office Animals Scientific Procedures Act, 1986 and United Kingdom Coordinating Committee on Cancer Research (UKCCCR) guidelines (23). Mice had access to food and water ad libitum. $5 \times 10^{6}$ SW1222 or LS174T cells were subcutaneously inoculated on the lower right flank of female CD1 nu/nu mice (0.1 mL per injection).

\section{In vivo experimental design}

Tumors were allowed to grow for 14-16 days, then randomly assigned to treatment and control groups. The treatment group received a single dose of ixazomib i.v., at $8,9.5$ or $11 \mathrm{mg} \mathrm{kg}^{-1}$. The control group received vehicle, consisting of the drug stock solution (2-Hydroxypropyl- $\beta$-cyclodextrin, Sigma Aldrich). Treated mice were maintained in a warmed cage using a thermostatic heating pad (Physitemp Instruments, Inc., New Jersey) and an infrared heating lamp (Zoo Med, UK) providing an ambient temperature of $24-26{ }^{\circ} \mathrm{C}$. Hydrogel was provided to prevent dehydration. Tumor volume $(V)$ was measured every 2 days using electronic calipers, according to (24) $V=w^{2} I / 2$, where $I$ and $w$ are the maximal tumor diameter and the diameter orthogonal to this measurement, respectively. The mass of each mouse was also measured using an electric balance (C-MAG HS 7 IKAMAG ${ }^{\circledR}$, Staufen, Germany).

Baseline MRI data were acquired 24 hours before drug administration (0 hours), with follow-up sessions at 48 and 72 hours. Immediately following the final MRI scan, mice were culled via cervical dislocation, tumors were resected and sent for ex vivo analysis.

\section{In vivo MRI protocol}

MRI data were acquired using a 9.4T scanner (Agilent Santa Clara, CA) with a $39 \mathrm{~mm}$ birdcage coil (Rapid MR International, Columbus, $\mathrm{OH})$. Mice were anaesthetized prior to and throughout each scanning session using isoflurane in $\mathrm{O}_{2}$ (2.5\% for induction, $1.25 \%-1.75 \%$ for maintenance). Core body temperature was maintained $37^{\circ} \mathrm{C}$ using a warm water heating system. Ventilation rate was monitored using a respiration pad (SA Instruments, USA) and maintained at $60-80$ breaths per minute by adjustment of isoflurane concentration.

Following shimming, a $\mathrm{T}_{2}$-weighted, fast spin echo sequence was used for tumor localization and volume measurement (see supplemental methods for details).

CEST MRI. Our CEST sequence was based on a gradient echo acquisition, and included the following parameters: repetition time (TR), $162 \mathrm{~ms}$; echo time (TE), $2 \mathrm{~ms}$; saturation power, $3 \mu \mathrm{T}$; flip angle, $20^{\circ}$; slice thickness, $1 \mathrm{~mm}$ (single slice); matrix size, 64×64; FOV, $30 \times 30 \mathrm{~mm}^{2}$. The slice was positioned to encompass the largest crosssectional area of each tumor. Sampling of each line of k-space was preceded by a train of three 50 ms Gaussian pulses to induce saturation. Each pulse had a flip angle of $20^{\circ}$, duration of 2 ms and with 2 ms spacing (25). Crusher gradients were applied between pulses to spoil any residual transverse magnetization. The sequence was repeated with saturation frequency offsets ranging from -6 to $6 \mathrm{ppm}$, at intervals of $0.12 \mathrm{ppm}$. A reference image was also acquired at an offset of $8000 \mathrm{ppm}\left(S_{0}\right)$. 
During post-processing, $z$-spectra were produced on a pixel-by-pixel basis, according to $Z(f)=S(f) / S_{0}$, where $f$ is the saturation offset frequency. These data were fitted with a summed Lorenzian model, with peak offsets corresponding to proton exchange between water and amide, amine and hydroxyl groups, alongside a broad peak corresponding to magnetization transfer (MT). Details of this modelling step are provided in the supplemental methods.

Diffusion MRI and relaxometry. Full details of diffusion $M R I, T_{1}$ and $T_{2}$ acquisition and quantification procedures are provided in the supplemental materials. In brief, diffusion MRI data were acquired using a multi-slice fast spin-echo sequence, with b-values ranging from 150 to $1070 \mathrm{~s} \mathrm{~mm}$. The apparent diffusion coefficient was quantified from these data by fitting to a simple exponential function (equ. 5). $T_{1}$ and $T_{2}$ were estimated from data acquired with a Look-Locker segmented inversion recovery sequence and a multi-echo multi-slice spin-echo sequence, respectively.

\section{Ex vivo analysis of resected tumor samples}

Resected tumors were cut in half: one half was flash-frozen in liquid nitrogen and stored at $-80^{\circ} \mathrm{C}$ (for Bradford Assay) and the other half was fixed in $5 \%$ formalin for immunohistochemical analysis.

Bradford Assay. Protein extraction reagent was added to the tumor samples at $1 \mathrm{~g} / 20 \mathrm{ml}$ and homogenized according to the manufacturer's instructions (T-PER ${ }^{\circledR}$ Tissue Protein Extraction Reagent, Thermo Scientific). The homogenized mixture was centrifuged at $10,000 \mathrm{~g}$ for five minutes to pellet tissue debris. The supernatant was collected or stored at $-20^{\circ} \mathrm{C}$ for Bradford assays. Bradford assays were carried out according to manufacturer's instructions (Pierce Coomassie Bradford Protein Assay Kit, Thermal Scientific).

Immunohistochemistry. GADD34 and cleaved caspase-3 immunohistochemistry was performed on all tumor samples (details provided in supplemental methods). All slides were haematoxylin counterstained.

\section{Statistical Analysis}

The median value for each imaging parameter was used for data analysis (rather than mean values, in order to limit the influence of extreme or outlier values resulting from convergence at local minima). Longitudinal data were normalised to the baseline measurement and expressed as a percentage change. Two-way analysis of variance between groups (ANOVA) (GraphPad Prism X.6.0.1, California, USA) was used for statistical analysis. Both the difference between control and treated groups at the same time points (between group analysis), and the difference between post-treatment and baseline data (within group analysis) were tested for statistical significance. $\mathrm{P}<0.05$ was considered significant. Correlations were assessed using Spearman's rho.

\section{Results}

\section{LS174T and SW1222 human colorectal cancer cell lines displayed differing in vitro sensitivities to ixazomib}

The MTT assay showed that both SW1222 and LS174T cell lines demonstrate a significant decrease in viability at doses greater than $12 \mathrm{nM}$ in SW1222 and 24 nM in LS174T cells (see Figure 1), at 24, 48 and 72 hours. Interestingly, both cell lines also exhibited a stimulatory response at low doses ( $<6$ nM in SW1222 and $<24$ nM in LS174T), evidenced by an increase in cell viability (130\% in SW1222 and 120\% in LS174T) that peaked at 48 hours. Overall, SW1222 cells were more sensitive to ixazomib than LS174T cells ( $/ C_{50}$ of $12.6 \mathrm{nM}$ and $41.1 \mathrm{nM}$ for SW1222 and LS174T, respectively), with an approximately three-fold lower viability at 48 hours than LS174T cells, 
and approximately ten-fold lower viability at 72 hours $\left(\mathrm{IC}_{50}\right.$ of $7.6 \mathrm{nM}$ and $78.4 \mathrm{nM}$ for SW1222 and LS174T, respectively).

\section{Ixazomib affected colorectal tumor xenograft growth in vivo, in a dose-dependent manner}

We next investigated the action of ixazomib in vivo, at 24 and 72 hours after dosing with ixazomib (at 11, 9.5 or $8 \mathrm{mg} \mathrm{kg}^{-1}$ ) or vehicle. MRI measurements of tumor volume confirmed that the growth of both SW1222 and LS174T tumors was significantly inhibited following ixazomib doses of 9.5 and $11 \mathrm{mg} \mathrm{kg}^{-1}$ relative to control tumors (Figs. 2 and S1). We also observed a small but significant increase in tumor volume at 72 hours (relative to baseline) at the lowest ixazomib dose $\left(8 \mathrm{mg} \mathrm{kg}^{-1}\right)$ in both SW1222 $(P<0.0001)$ and LS174T $(P<0.001)$ tumors, which potentially mirrors the low-dose stimulatory effect that were observed in in vitro experiments.

\section{CEST MRI measurements reflected response to proteasome inhibition in colorectal cancer xenografts}

CEST MRI measurements were represented using Z-spectra, a plot of normalized water signal as a function of saturation frequency (Figure 3a). Amide, amine and hydroxyl groups are located at approximately 3.5, 2.4 and $1.2 \mathrm{ppm}$ from water, respectively (25-27), alongside contributions from magnetization transfer (MT, $-2.4 \mathrm{ppm}$ (26)), relayed nuclear Overhouser effect (NOE, $-3.3 \mathrm{ppm}(28)$ ) and lipids (-1 to $-5 \mathrm{ppm}$ (27)) combined into a single, broad peak. Fitting our summed Lorentzian model on a pixel-by-pixel basis allowed images of each z-spectrum contribution to be generated (Figure 3b).

The area under amide and amine peaks significantly increased following ixazomib treatment, relative to pretreatment measurements (Figs. 4 and S1). A summary of all significant changes, for each tumor type, ixazomib dose and time post-dosing is provided in Table $\mathbf{2}$. These data, in combination, demonstrate the smaller magnitude of change in parameter values following treatment in LS174T tumors compared with SW1222 tumors, potentially reflecting their lower sensitivity to ixazomib.

\section{Changes in amide and amine peaks were significantly correlated with ixazomib dose}

A significant correlation was found between amide peak area and ixazomib dose in SW1222 tumors at 72 hours $(r=0.98, P<0.05)$ (Figure 4). This was also reflected in the change in the amine area of the $Z$ spectrum, with a strong positive correlation between the dose concentration and amine signals $(r=0.97, P<0.05)$. Conversely, no parameters in LS174T tumors exhibited a dose-dependent response, which could reflect their reduced sensitivity to ixazomib, compared with SW1222 tumors.

\section{Gross tissue protein measurements from Bradford assay displayed a complex relationship with ixazomib dose}

Bradford assay at 72 hours showed a significant difference in mean protein concentration between SW1222 and LS174T control tumors $\left(1386 \mu \mathrm{g} \mathrm{ml}^{-1}, \mathrm{P}<0.001\right)$. LS174T tumors had a small but significantly lower protein concentration than control tumors when dosed at $8 \mathrm{mg} \mathrm{kg}^{-1}$, whereas higher doses did not induce a significant change (Figure 5c). SW1222 tumors exhibited lower gross protein concentration when treated at 8 and $11 \mathrm{mg}$ $\mathrm{kg}^{-1}$, compared with control tumors, but a significantly higher protein concentration at $9.5 \mathrm{mg} \mathrm{kg}^{-1}$.

No significant correlation was found between Bradford assay results and in vivo amide and amine peak area $(P>0.05$, Spearman's rho). Equally, no significant differences were found in baseline CEST parameters, between tumors, that might have reflected the difference found between tumor types in Bradford assay results. 


\section{Diffusion MRI measures reflected changes in CEST parameters, but $T_{1}$ and $T_{2}$ showed mixed changes}

A significant increase was found in ADC from baseline, in SW1222 tumors at 72 hours, for ixazomib doses of 9.5 and $11 \mathrm{mg} \mathrm{kg}^{-1}$ (41.5\%, $\mathrm{P}<0.01$ and $22.1 \%, \mathrm{P}<0.05$, respectively), which were significantly greater than control tumors (Figure 4 and Table 2). The change in ADC from baseline was also significantly correlated with ixazomib dose $(\mathrm{P}<0.01)$. In LS174T tumors, only the $9.5 \mathrm{mg} \mathrm{kg}^{-1}$ group showed a significant increase, when compared against baseline $(55.19 \%, \mathrm{P}<0.001)$ and control tumors $(46.92 \%, \mathrm{P}<0.05)$.

$T_{1}$ and $T_{2}$ showed a more mixed set of changes in both tumor types, with fewer time points achieving significance (see Figure 4 and Table 2). $T_{1}$ and $T_{2}$ showed both increases and decreases over time, and did not display a simple relationship with ixazomib dose, other than for the change in $T_{2}$ in SW1222 tumors at 72 hours, which was directly correlated with dose $(r=0.99, P<0.01)$.

\section{Immunohistochemical markers of apoptosis significantly correlated with ixazomib dose in SW1222, but not LS174T tumors}

To evaluate the relationship between CEST imaging biomarkers and physiological changes caused by proteasome inhibition, we used immunohistochemistry to measure the percentage expression of cell death factor, caspase 3 , and $\operatorname{GADD} 34(20,29)$. Our results revealed significantly higher caspase 3 expression in SW1222 tumors for doses of $9.5 \mathrm{mg} \mathrm{kg}^{-1}(25.61 \%, \mathrm{P}<0.05)$ and $11 \mathrm{mg} \mathrm{kg}^{-1}(29.87 \%, \mathrm{P}=0.002)$, relative to controls (Figure 5). Caspase 3 expression was also significantly correlated with ixazomib dose $(r=0.95, P<0.05)$. Though small, there was significantly lower caspase 3 expression in LS174T tumors for a dose of $8 \mathrm{mg} \mathrm{kg}^{-1}(4.57 \%, P=0.0127)$, relative to controls, but no significant differences at higher doses. Whilst there was no significant change in GADD34 percentage expression relative to controls in either SW1222 or LS174T tumors (Figure 5), as for caspase 3, a significant correlation was found between GADD34 expression with increasing dosing concentration in SW1222 tumors $(r=0.96, P<0.05)$.

\section{Discussion}

Noninvasive imaging biomarkers for cancer therapy are critically needed in the clinic, particularly those that can detect a functional response prior to much slower changes in tumor volume (22). CEST is a noninvasive imaging technique, based on MRI, in which image contrast is induced via the exchange of protons between water and chemical groups such as amides, amines and hydroxyls (17). The presence of amide groups in protein backbones and amine groups on peptides make CEST sensitive to changes in the concentration of both types of molecules (30-34), and therefore as a potential biomarker of disrupted protein homeostasis. We therefore aimed to evaluate the change in CEST MRI parameters (alongside other more conventional MRI measures) following dosing with ixazomib, in two colorectal carcinoma tumor xenograft models (SW1222 and LS174T).

Our experiments showed that ixazomib elicited a significant growth-inhibitory effect in both colorectal cell lines, as evidenced by a reduction in cell viability in vitro and in the reduction of tumor growth rates in vivo. SW1222 tumors were found to be more sensitive to ixazomib than LS174T tumors, which could be due to their different K-Ras mutations: SW1222 cells have a K-Ras mutation at A146V (35), compared with G12D for LS174T tumors (36), which has been proposed to infer a greater sensitivity to proteosome inhibition (11). In addition, the microenvironment of the two tumor types could provide a source of resistance in vivo, with LS174T tumors being less vascular, less differentiated and less perfused than SW1222 tumors (37-39). 
Our CEST measurements showed a significant increase in amide, amine and $\mathrm{OH}$ peak areas with time following ixazomib treatment, with the greatest effects observed at 72 hours following dosing. Moreover, several parameters varied in a dose-dependent manner, with a significant correlation measured between amide and amine peak size and ixazomib dose in SW1222 tumors. This reflected similar trends in our immunohistochemical measurements of caspase 3 and GADD34 expression, which were themselves consistent with previous studies $(20,29)$. This induction of apoptosis could also explain the observed increases in ADC, in which changes in cell size and the induction of micro-necrosis causes less restricted water diffusion (37).

However, the relationship between CEST parameters and Bradford assay were less straightforward to interpret, with no apparent correspondence evident between the two measurement types. Several factors might help to explain this disparity. Firstly, CEST MRI is sensitive to mobile protein and peptides, whereas the Bradford assay is unaffected by amino acids or peptides smaller than $3 \mathrm{kDa}$ (according to manufacturer's instructions). Secondly, CEST contrast is dependent on several other parameters including $T_{1}, T_{2}$ and $\mathrm{pH}$, which were not controlled for in this study. $T_{1}$ and $T_{2}$ were found to show few consistent changes when measured directly, so it is arguable that these were unlikely to have influenced CEST measurements significantly. The pH of tumor tissue could also have influenced our measurements, and a fully quantitative model (26) could potentially be implemented to separate proton exchange rate and pool sizes.

Interestingly, we found evidence that ixazomib causes a mild stimulatory effect at low doses (40). This bi-phasic effect has been previously observed in a wide range of anticancer agents (40-43) alongside physical interventions such as radiotherapy (44). In vitro MTT assays revealed increased cell viability at low doses, and tumor growth was slightly enhanced at an in vivo dose of $8 \mathrm{mg} \mathrm{kg}^{-1}$. Likewise, caspase 3 expression was slightly decreased, relative to controls, in LS174T tumors dosed at $8 \mathrm{mg} \mathrm{kg}^{-1}$. Whether this effect was reflected in MRI measurements is unclear, as no significant changes were observed in CEST parameters (amide and amine areas) at low ixazomib doses. However, this effect warrants further investigation, particularly if the treatment of tumors with poor delivery profiles (for example, pancreatic adenocarcinoma) is currently under consideration, as delivered doses might be significantly lower than expected. In this context, in particular, amide and amine CEST signals and ADC could find strong utility, alongside complementary measures of blood flow and interstitial transport.

A key question is whether these findings can be translated and reproduced in humans. Clinical MRI is typically undertaken at a field strength of 3T, which is lower than the 9.4T system used here. Translation between field strengths presents several challenges (such as reduced separation between exchange peaks, lower signalto-noise ratios, and slower relaxation rates), but which have been overcome in other translational studies. Equally, the evaluation of Ixazomib in human solid tumors is at an early stage and the data presented here demonstrates its efficacy in two colorectal carcinoma xenograft models. It also identifies several promising, noninvasive biomarkers (amide and amine CEST signals and ADC) that could be explored for determining early treatment response and detecting disrupted protein homeostasis. In addition, they could be further evaluated in the assessment of plasma cell malignancies (currently the predominant targets of Ixazomib), with a view to reducing the need for invasive bone marrow aspirates or biopsy. 


\section{References}

1. Douglas PM, Dillin A. Protein homeostasis and aging in neurodegeneration. J Cell Biol. 2010;190(5):719-

29.

2. Chiti F, Dobson CM. Protein misfolding, functional amyloid, and human disease. Annu Rev Biochem. 2006;75:333-66.

3. Powers ET, Morimoto RI, Dillin A, Kelly JW, Balch WE. Biological and chemical approaches to diseases of proteostasis deficiency. Annual review of biochemistry. 2009;78:959-91.

4. Manasanch EE, Orlowski RZ. Proteasome inhibitors in cancer therapy. Nature reviews Clinical oncology. 2017.

5. Antonia Field-Smith GJM, and Faith E Davies. Bortezomib (Velcade ${ }^{\mathrm{TM}}$ ) in the Treatment of Multiple Myeloma. Ther Clin Risk Manag. 2006;2(3):271-9.

6. Buac D, Shen M, Schmitt S, Kona FR, Deshmukh R, Zhang Z, et al. From Bortezomib to other Inhibitors of the Proteasome and Beyond. Curr Pharm Design. 2013;19(22):4025-38.

7. Paramore A, Frantz S. Bortezomib. Nat Rev Drug Discov. 2003;2(8):611-2.

8. Muz B, Ghazarian RN, Ou M, Luderer MJ, Kusdono HD, Azab AK. Spotlight on ixazomib: potential in the treatment of multiple myeloma. Drug design, development and therapy. 2016;10:217-26.

9. Raedler LA. Ninlaro (Ixazomib): First Oral Proteasome Inhibitor Approved for the Treatment of Patients with Relapsed or Refractory Multiple Myeloma. Am Health Drug Benefits. 2016;9(Spec Feature):102-5.

10. Al-Salama ZT, Garnock-Jones KP, Scott LJ. Ixazomib: A Review in Relapsed and/or Refractory Multiple Myeloma. Targeted oncology. 2017.

11. Chattopadhyay N, Berger AJ, Koenig E, Bannerman B, Garnsey J, Bernard H, et al. KRAS Genotype Correlates with Proteasome Inhibitor Ixazomib Activity in Preclinical In Vivo Models of Colon and Non-Small Cell Lung Cancer: Potential Role of Tumor Metabolism. PloS one. 2015;10(12):e0144825.

12. Li H, Chen Z, Hu T, Wang L, Yu Y, Zhao Y, et al. Novel proteasome inhibitor ixazomib sensitizes neuroblastoma cells to doxorubicin treatment. Scientific reports. 2016;6:34397.

13. Yang LF, Wan JF, Xiao S, Barkhouse D, Zhu J, Li GC, et al. BH3 mimetic ABT-737 sensitizes colorectal cancer cells to ixazomib through MCL-1 downregulation and autophagy inhibition. Am J Cancer Res. 2016;6(6):1345-+.

14. Jia G, Abaza R, Williams JD, Zynger DL, Zhou J, Shah ZK, et al. Amide proton transfer MR imaging of prostate cancer: a preliminary study. J Magn Reson Imaging. 2011;33(3):647-54.

15. Hong XH, Liu L, Wang MY, Ding K, Fan Y, Ma B, et al. Quantitative multiparametric MRI assessment of glioma response to radiotherapy in a rat model. Neuro-Oncology. 2014;16(6):856-67.

16. Zhou J, Tryggestad E, Wen Z, Lal B, Zhou T, Grossman R, et al. Differentiation between glioma and radiation necrosis using molecular magnetic resonance imaging of endogenous proteins and peptides. Nature Medicine. 2011;17:130-4.

17. van Zijl PCM, Yadav NN. Chemical Exchange Saturation Transfer (CEST): What is in a Name and What Isn't? Magnetic resonance in medicine. 2011;65(4):927-48.

18. Crawford LJ, Walker B, Irvine AE. Proteasome inhibitors in cancer therapy. J Cell Commun Signal. 2011;5(2):101-10.

19. Romaniuk W, Oldziej AE, Zinczuk J, Kloczko J. Proteasome inhibitors in cancer therapy. Postep Hig Med Dosw. 2015;69:1443-50.

20. Chauhan D, Tian Z, Zhou B, Kuhn D, Orlowski R, Raje N, et al. In Vitro and In Vivo Selective Antitumor Activity of a Novel Orally Bioavailable Proteasome Inhibitor MLN9708 against Multiple Myeloma Cells. Clin Cancer Res. 2011;17(16):5311-21.

21. Kupperman E, Lee EC, Cao Y, Bannerman B, Fitzgerald M, Berger A, et al. Evaluation of the proteasome inhibitor MLN9708 in preclinical models of human cancer. Cancer Res. 2010;70(5):1970-80.

22. O'Connor JP, Aboagye EO, Adams JE, Aerts HJ, Barrington SF, Beer AJ, et al. Imaging biomarker roadmap for cancer studies. Nature reviews Clinical oncology. 2017;14(3):169-86.

23. Workman P, Aboagye EO, Balkwill F, Balmain A, Bruder G, Chaplin DJ, et al. Guidelines for the welfare and use of animals in cancer research. Brit J Cancer. 2010;102(11):1555-77. 
24. Williamson MJ SM, Terkelsen J, Robertson R, Yu L, Xia C, Hatsis P, Bannerman B, Babcock T, Cao Y, Kupperman $\mathrm{E}$. The relationship among tumor architecture, pharmacokinetics, pharmacodynamics, and efficacy of bortezomib in mouse xenograft models. Mol Cancer Ther. 2009;8(12):3234-43.

25. Walker-Samuel S, Ramasawmy R, Torrealdea F, Rega M, Rajkumar V, Johnson SP, et al. In vivo imaging of glucose uptake and metabolism in tumors. Nature medicine. 2013;19(8):1067-72.

26. Chappell MA, Donahue MJ, Tee YK, Khrapitchev AA, Sibson NR, Jezzard P, et al. Quantitative Bayesian model-based analysis of amide proton transfer MRI. Magn Reson Med. 2013;70(2):556-67.

27. Walker-Samuel S, Johnson SP, Pedley B, Lythgoe MF, Golay X. Extracranial measurements of amide proton transfer using exchange-modulated point-resolved spectroscopy (EXPRESS). NMR Biomed. 2012;25(6):829-34.

28. Jones CK, Huang A, Xu J, Edden RA, Schar M, Hua J, et al. Nuclear Overhauser enhancement (NOE) imaging in the human brain at 7T. Neurolmage. 2013;77:114-24.

29. Kupperman E, Lee EC, Cao Y, Bannerman B, Fitzgerald M, Berger A, et al. Evaluation of the Proteasome Inhibitor MLN9708 in Preclinical Models of Human Cancer (vol 70, pg 1970, 2010). Cancer Res. 2010;70(9).

30. Zhou JY, Lal B, Wilson DA, Laterra J, van Zijl PCM. Amide proton transfer (APT) contrast for imaging of brain tumors. Magnetic resonance in medicine. 2003;50(6):1120-6.

31. Salhotra A, Lal B, Laterra J, Sun PZ, van Zijl PC, Zhou J. Amide proton transfer imaging of 9 L gliosarcoma and human glioblastoma xenografts. NMR Biomed. 2008;21(5):489-97.

32. Jones CK, Schlosser MJ, van Zijl PCM, Pomper MG, Golay X, Zhou JY. Amide proton transfer imaging of human brain tumors at 3T. Magnetic resonance in medicine. 2006;56(3):585-92.

33. Dula AN, Arlinghaus LR, Dortch RD, Dewey BE, Whisenant JG, Ayers GD, et al. Amide proton transfer imaging of the breast at $3 \mathrm{~T}$ : Establishing reproducibility and possible feasibility assessing chemotherapy response. Magnetic resonance in medicine. 2013;70(1):216-24.

34. Yuan J CS, King AD, Zhou J, Bhatia KS, Zhang Q, Yeung DK, Wei J, Mok GS, Wang YX. Amide proton transfer-weighted imaging of the head and neck at $3 \mathrm{~T}$ : a feasibility study on healthy human subjects and patients with head and neck cancer. NMR Biomed. 2014;27(10):1239-4.

35. Janakiraman M, Vakiani E, Zeng Z, Pratilas CA, Taylor BS, Chitale D, et al. Genomic and biological characterization of exon 4 KRAS mutations in human cancer. Cancer Res. 2010;70(14):5901-11.

36. Kato J, Futamura M, Kanematsu M, Gaowa S, Mori R, Tanahashi T, et al. Combination therapy with zoledronic acid and cetuximab effectively suppresses growth of colorectal cancer cells regardless of KRAS status. International journal of cancer. 2016;138(6):1516-27.

37. Panagiotaki E, Walker-Samuel S, Siow B, Johnson SP, Rajkumar V, Pedley RB, et al. Noninvasive quantification of solid tumor microstructure using VERDICT MRI. Cancer research. 2014;74(7):1902-12.

38. Dearling JL, Flynn AA, Qureshi U, Whiting S, Boxer GM, Green A, et al. Localization of radiolabeled antiCEA antibody in subcutaneous and intrahepatic colorectal xenografts: influence of tumor size and location within host organ on antibody uptake. Nucl Med Biol. 2009;36(8):883-94.

39. Folarin AA, Konerding MA, Timonen J, Nagl S, Pedley RB. Three-dimensional analysis of tumour vascular corrosion casts using stereoimaging and micro-computed tomography. Microvascular research. 2010;80(1):8998.

40. Calabrese EJ. Cancer biology and hormesis: human tumor cell lines commonly display hormetic (biphasic) dose responses. Critical reviews in toxicology. 2005;35(6):463-582.

41. Gaya A, Akle CA, Mudan S, Grange J. The Concept of Hormesis in Cancer Therapy - Is Less More? Cureus. 2015;7(4):e261.

42. Azam F, Mehta S, Harris AL. Mechanisms of resistance to antiangiogenesis therapy. Eur J Cancer. 2010;46(8):1323-32.

43. Reynolds AR. Potential relevance of bell-shaped and u-shaped dose-responses for the therapeutic targeting of angiogenesis in cancer. Dose Response. 2010;8(3):253-84.

44. Vaiserman AM. Radiation hormesis: historical perspective and implications for low-dose cancer risk assessment. Dose Response. 2010;8(2):172-91. 


\section{Tables}

\begin{tabular}{|l|l|l|l|}
\hline \multirow{2}{*}{ Pool (index) } & \multicolumn{3}{|l|}{ Gaussian prior parameters: mean (s.d.) } \\
\cline { 2 - 3 } & $\boldsymbol{b}$ (ms) & $\boldsymbol{a}$ (ppm) & \multicolumn{1}{|c|}{$\boldsymbol{f}_{\text {o }}$ (ppm) } \\
\hline Water (0) & $5(3)$ & $\mathrm{n} / \mathrm{a}$ & \multirow{2}{*}{$0(3)$} \\
\hline Amide (1) & $8(5)$ & $3.5(0.6)$ & \\
\hline Amine (2) & $8(5)$ & $2.4(0.6)$ & \\
\hline Hydroxyl (3) & $5(5)$ & $1.2(0.6)$ & \\
\hline MT \& NOE (4) & $0.1(1)$ & $-2.4(0.6)$ & \\
\hline
\end{tabular}

Table 1. Gaussian prior distribution parameter values for the maximum likelihood model, expressed as mean (s.d.) (derived from Chappell et al. (26)). Entries marked with 'n/a' were fixed (see text for details).

\begin{tabular}{|c|c|c|c|c|c|c|}
\hline \multicolumn{7}{|c|}{ SW1222 } \\
\hline Time post-dosing & \multicolumn{3}{|c|}{24 hours } & \multicolumn{3}{|c|}{72 hours } \\
\hline Dose $\left(\mathrm{mg} \mathrm{kg}^{-1}\right)$ & 8 & 9.5 & 11 & 8 & 9.5 & 11 \\
\hline \multicolumn{7}{|l|}{ Amide (peak area) } \\
\hline \multicolumn{7}{|l|}{ Amine (peak area) } \\
\hline \multicolumn{7}{|l|}{$\mathrm{OH}$ (peak area) } \\
\hline \multicolumn{7}{|l|}{ MT (peak area) } \\
\hline \multicolumn{7}{|l|}{$A D C$} \\
\hline \multicolumn{7}{|l|}{$\mathrm{T}_{1}$} \\
\hline \multicolumn{7}{|l|}{$\mathrm{T}_{2}$} \\
\hline \multicolumn{7}{|l|}{ Bradford assay } \\
\hline \multicolumn{7}{|l|}{ Caspase 3} \\
\hline \multirow{2}{*}{\multicolumn{7}{|c|}{ GADD34 }} \\
\hline & & & & & & \\
\hline Time post-dosing & \multicolumn{3}{|c|}{24 hours } & \multicolumn{3}{|c|}{72 hours } \\
\hline Dose $\left(\mathrm{mg} \mathrm{kg}^{-1}\right)$ & 8 & 9.5 & 11 & 8 & 9.5 & 11 \\
\hline \multicolumn{7}{|l|}{ Amide (peak area) } \\
\hline \multicolumn{7}{|l|}{ Amine (peak area) } \\
\hline \multicolumn{7}{|l|}{$\mathrm{OH}$ (peak area) } \\
\hline \multicolumn{7}{|l|}{ MT (peak area) } \\
\hline \multicolumn{7}{|l|}{$A D C$} \\
\hline \multicolumn{7}{|l|}{$\mathrm{T}_{1}$} \\
\hline \multicolumn{7}{|l|}{$\mathrm{T}_{2}$} \\
\hline \multicolumn{7}{|l|}{ Bradford assay } \\
\hline \multicolumn{7}{|l|}{ Caspase 3} \\
\hline GADD34 & & & & & & \\
\hline
\end{tabular}

Table 2. Summary of significant changes in each quantitative parameter measured, relative to controls. Red cells indicate a significant increase, blue cells a significant decrease, white cells no significant change, and grey cells show where no data were available. 


\section{Figures}

a

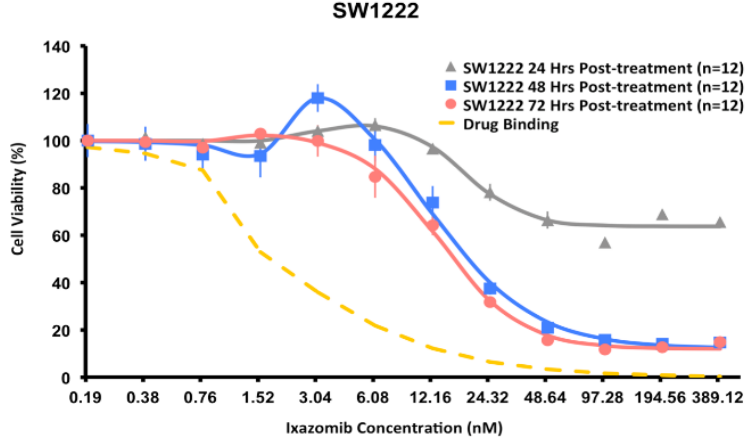

b

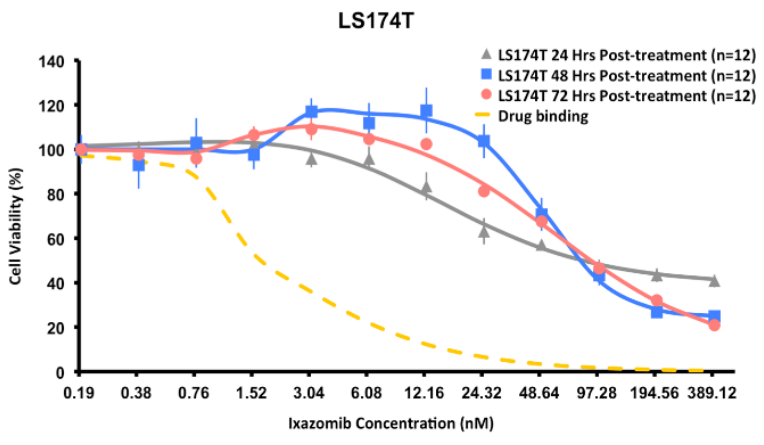

Figure 1. Dose-response curves showing cell viability as a function of ixazomib concentration, measured with the MTT assay, at 24, 48 and 72 hours post-dosing, in SW1222 (a) and LS174T (b) cells (data points are mean \pm standard error). These in vitro results show that cell viability decreases at higher ixazomib doses ( $>12 \mathrm{nM}$ in SW1222 and $>24 \mathrm{nM}$ in LS174T) and that growth is stimulated at lower doses ( $<6 \mathrm{nM}$ in SW1222 and <24 nM in LS174T). SW1222 cells are more sensitive to ixazomib than LS174T cells, which is in keeping with their K-Ras mutation status. Dashed lines show ixazomib binding estimates. Data points are mean \pm standard error.

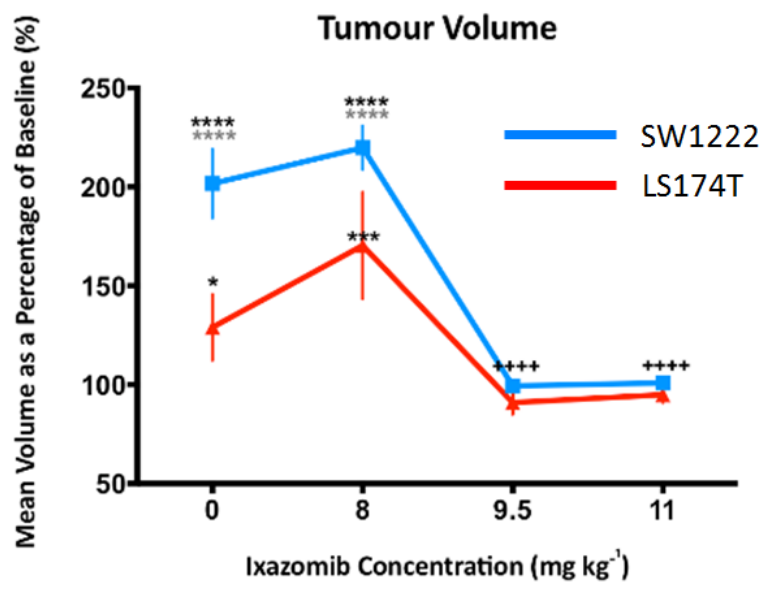

Figure 2. Mean relative change in tumour volume as a function of ixazomib dose, at 72 hours postdosing, in LS174T and SW1222 human colorectal carcinoma mouse xenografts. Tumour growth was significantly inhibited at the two highest doses investigated ( 9.5 and $11 \mathrm{mg} \mathrm{kg}^{-1}$ ), and mildly (but significantly) stimulated at the lowest dose $\left(8 \mathrm{mg} \mathrm{kg}^{-1}\right)$. Tumour volumes were measured using volumetric MRI, and error bars represent the standard error in each measurement; * $\mathrm{P}<0.05$, ** $\mathrm{P}<0.01,{ }^{* * *} \mathrm{P}<0.001, * * * * \mathrm{P}<0.0001$ (two-way ANOVA); *(black) = compared to baseline (pre-dosing) measurement, ${ }^{*}($ grey $)=$ compared to measurement at 24 hours after ixazomib dose, ${ }^{+}=$compared to control measurement. 
a
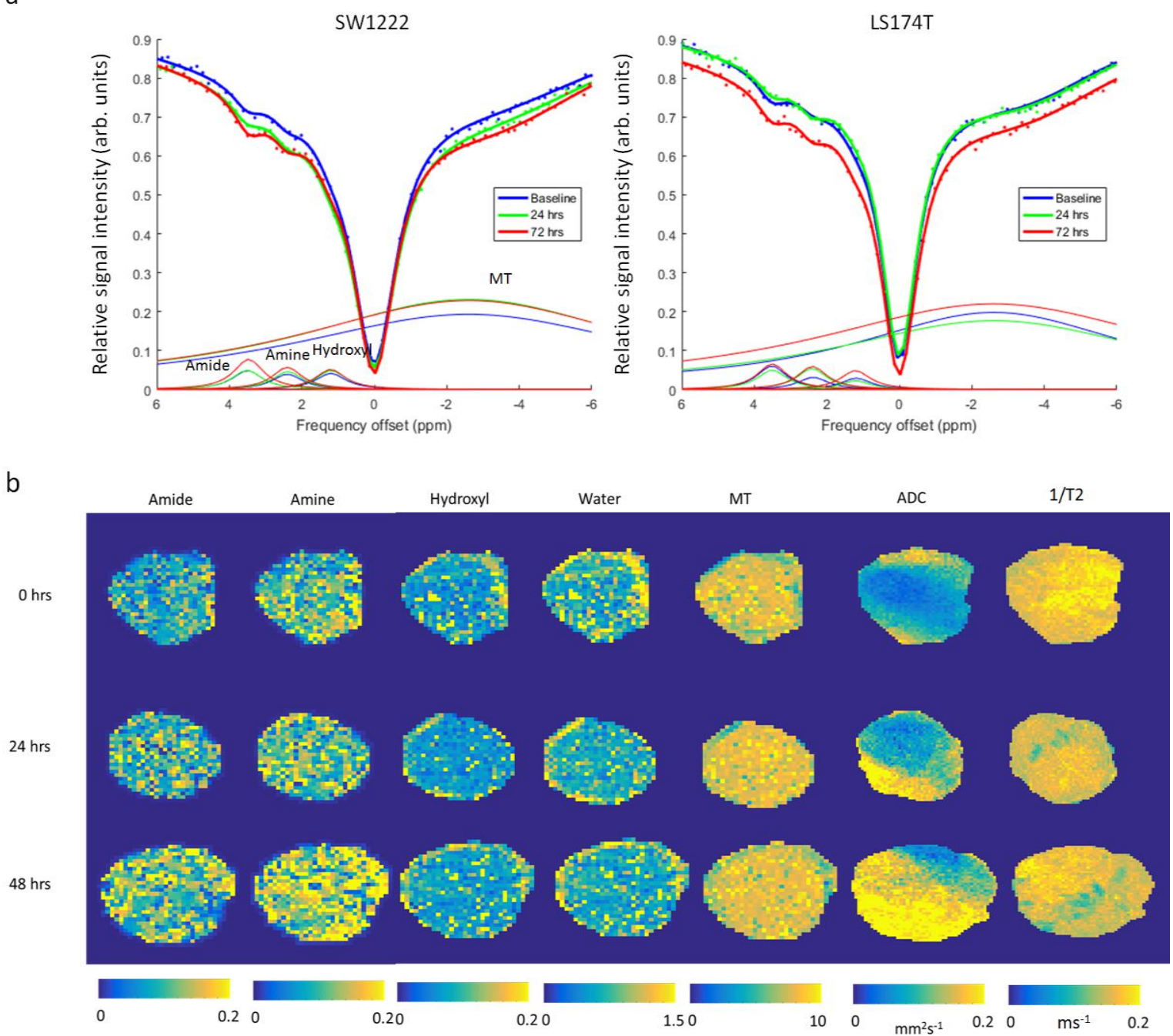

Figure 3. Example MRI measurements in SW1222 and LS174T tumours, prior to and at 24 and 72 hours following treatment with ixazomib, showing increasing amide and amine peak areas, alongside increasing apparent diffusion coefficient (ADC). (a) Z-spectra from CEST MRI, showing the normalised MRI signal intensity as a function of saturation frequency, averaged over a single representative tumour dataset at baseline and 24 and 72 hours following dosing with $11 \mathrm{mg} \mathrm{kg}^{-1}$ of ixazomib. Data points were fitted to a Bayesian model that allowed the individual influence of proton exchange between water and amide, amine and hydroxyl groups to be isolated (1.2, 2.4 and 3.5 ppm, respectively), alongside magnetisation transfer (MT, -2.4 ppm). Water peaks $(0 \mathrm{ppm})$ have been removed for clarity. (b) Images of amide, amine hydroxyl, MT and water peak areas, acquired noninvasively in an example SW1222 tumour at baseline and post-therapy (24 and 72 hours). Maps of the apparent diffusion coefficient (ADC) from diffusion MRI measurements and the transverse relaxation time $\left(T_{2}\right)$ are also shown. 

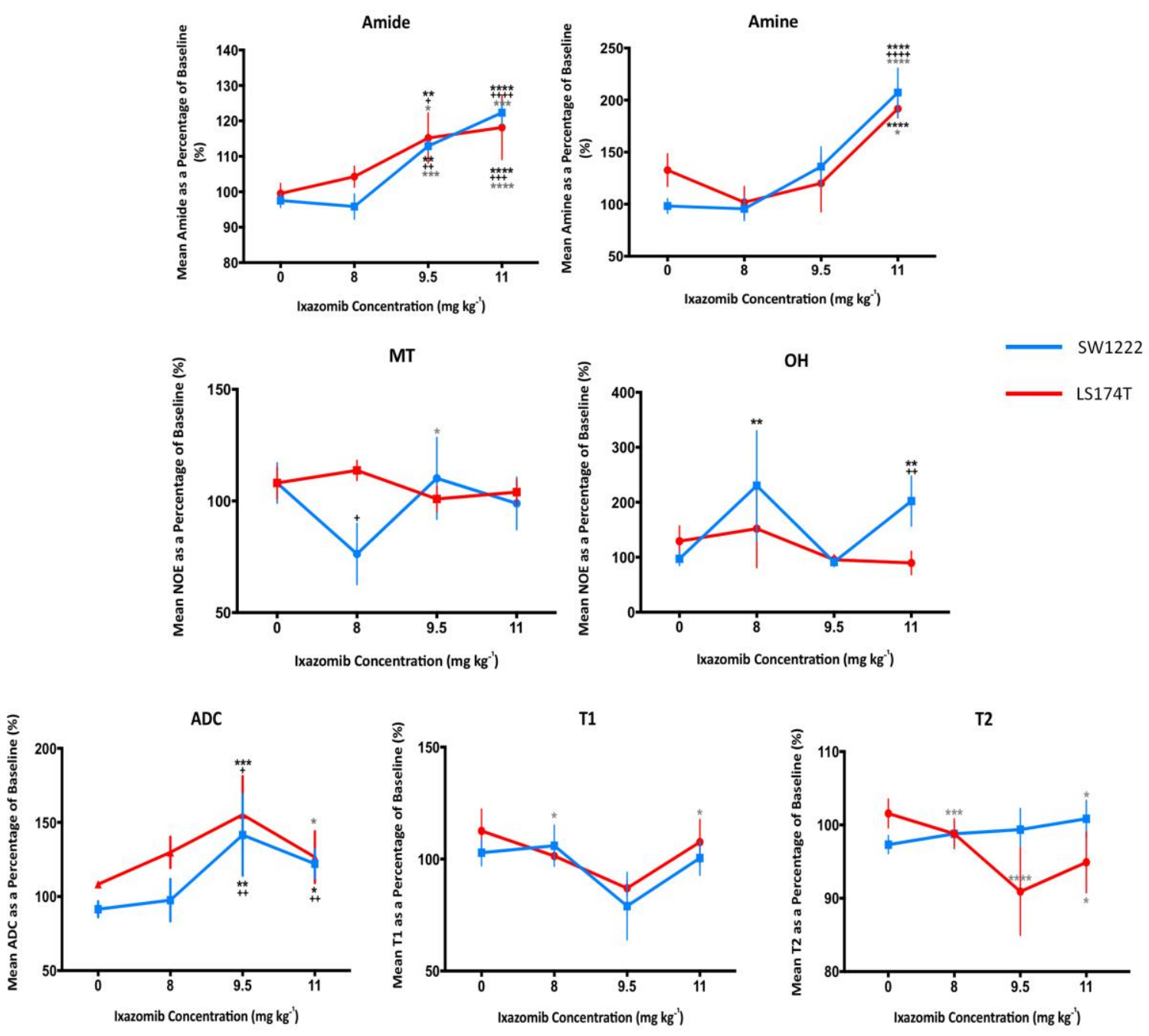

Figure 4. Summary of changes in quantitative, non-invasive MRI parameters, as a function of ixazomib dose $\left(8,9.5\right.$ and $\left.11 \mathrm{mg} \mathrm{kg}^{-1}\right)$, at 72 hours post-dosing. Only CEST measurements associated with amines and amide groups show a correspondence with ixazomib dose. Significant increases, in a dose-dependent manner, were observed in amine and amide peak areas from CEST MRI measurements. Tumour apparent diffusion coefficient (ADC) also increased significantly for the two highest doses, and $T_{2}$ decreased significantly in LS174T tumours only. Data points are mean \pm standard error; ${ }^{*} \mathrm{P}<0.05,{ }^{* *} \mathrm{P}<0.01,{ }^{* * *} \mathrm{P}<0.001, * * * * \mathrm{P}<0.0001$ (twoway ANOVA); ${ }^{*}($ black $)=$ compared to baseline, ${ }^{*}($ grey $)=$ compared to 24 hours post-ixazomib dose ${ }^{+}=$ compared to control. 
a Caspase 3

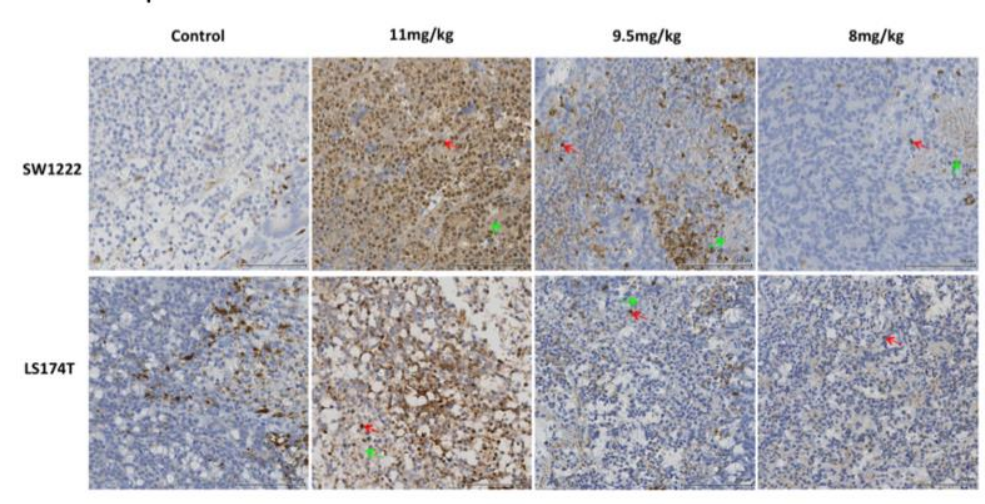

b GADD34

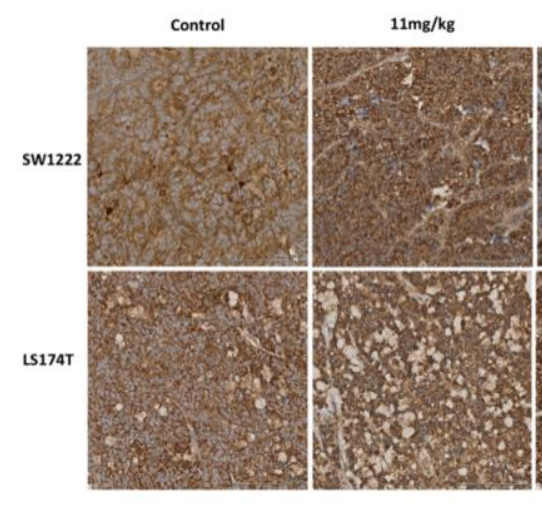

$9.5 \mathrm{mg} / \mathrm{kg}$

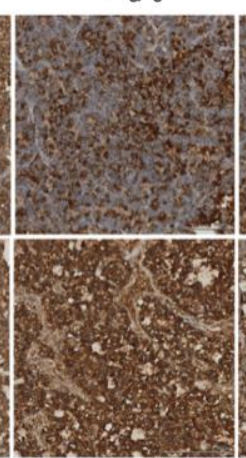

C

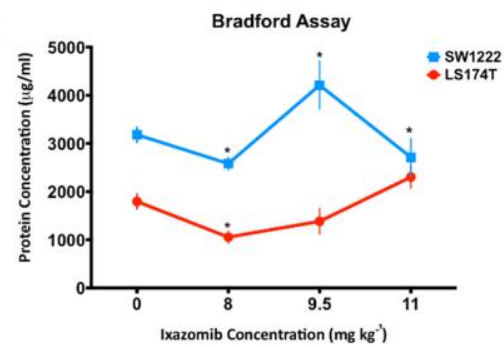

d

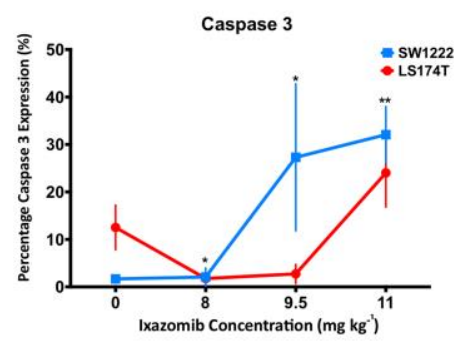

e

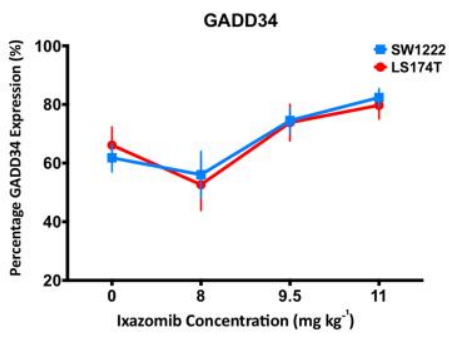

Figure 5. Ex vivo analysis of resected tumour tissue. Images of (a) caspase 3 and (b) GADD34 expression at 72 hours following dosing with vehicle, $8 \mathrm{mg} \mathrm{kg}^{-1}, 9.5 \mathrm{mg} \mathrm{kg}^{-1}$ or $11 \mathrm{mg} \mathrm{kg}^{-1}$. Caspase 3-positive nuclei are stained brown (as labelled with a red arrow) and blue otherwise (haematoxylin), and example necrotic areas are labelled with a green arrow. GADD34-positive areas are stained brown and nuclei are counter-stained in blue with haematoxylin. (c) The results of Bradford assay, to measure gross tissue protein. Summary data from quantitative analysis of immunohistochemistry images are shown for caspase 3 (d) and GADD34 (e), for each study group. Data points are mean \pm standard error $\left({ }^{*} \mathrm{P}<0.05\right.$, ${ }^{* *} \mathrm{P}<0.01$, ordinary one-way ANOVA of MannWhitney test). 


\section{Supplemental methods}

\section{Cell culture}

SW1222 and LS174T cells were cultured in minimum essential medium eagle (MEME, M4655, SigmaAldrich Cell Culture, Gillingham, UK) with 5\% fetal bovine serum. On the day of inoculation, the cells were harvested by washing once in phosphate buffered saline (PBS) solution (Sigma-Aldrich Cell Culture, Gillingham, UK) and trypsinised at $37^{\circ} \mathrm{C}$ for 5 minutes. The cells were pelleted by suspending in MEME and centrifuged at $1600 \times \mathrm{g}$ for 5 minutes. The suspension mixture was removed and the pelleted cells were washed in PBS and then in serum-free media at $1600 \mathrm{x} g$ for 5 minutes. Before the final wash in serum-free medium, $10 \mu l$ of the cell suspension mixed with trypan blue (Invitrogen) was transferred to a cell counting chamber slide (Invitrogen) and cells were counted with a Countess Automated Cell Counter (Invitrogen). The cells pelleted after the final wash were suspended in known volume serum free-media.

\section{MTT assay}

Labeling. Ixazomib was diluted from $10 \mathrm{mM}$ stock solution in complete media from 400 to $1.6 \mathrm{nM}$ using 2 -fold serial dilution. Media in each well was removed and replaced with $90 \mu \mathrm{L}$ of ixazomib, with corresponding concentration. Cells were left for 24,48 or 72 hours, then labeled with $10 \mu \mathrm{L}$ of the $12 \mathrm{mM}$ MTT reagent, and incubated for 2 hours at $37^{\circ} \mathrm{C}$. After the incubation period, the media was removed and replaced with $100 \mu \mathrm{L}$ of DMSO per well, and mixed thoroughly using an orbital plate shaker for 2 minutes. The microplate was incubated for $37^{\circ} \mathrm{C}$ for $10 \mathrm{~min}$ and were read using a microplate absorbance reader at $540 \mathrm{~nm}$.

Quantification. We estimated cell viability from the absorbance measure (unitless), in which the viability of treated cells was expressed as a percentage of the mean absorbance of control cells. Mean measurements of cell viability, expressed as a percentage change from baseline, were fitted to the modified Hill equation:

$$
y=m+\frac{p_{1}-m}{\left(1+\frac{E C_{50}}{[I]}\right)^{n H_{1}}}+\frac{p p_{2}-m}{\left(1+\frac{[I]}{I C_{50}}\right)^{n H H_{2}}}
$$

where $m$ is the maximum percentage viability; $p_{1}$ is the viability at low concentration (constrained to $100 \%)$, and $p_{2}$ is the viability at higher concentrations; $[I]$ is the concentration of ixazomib (nM); $E C_{50}$ and $I C_{50}$ are concentrations that produced half-maximal stimulatory and inhibitory effects (nM), respectively; $n H_{1}$ and $n H_{2}$ are unitless Hill factors for stimulatory and inhibitory slopes, respectively.

The modified Hill equation describes the binding of a drug to a receptor, with an empirical modification factor $(n H)(1)$. In our implementation, we modeled a single ixazomib binding site on the proteasome. The relationship between binding the proteasome and inhibition of cell proliferation is unknown. The data were fitted with a two-component Hill Equation to estimate the $I C_{50}$ concentration and maximum effect. Data were fitted using Prism (GraphPad Prism X.6.0.1, California, USA).

\section{CEST z-spectrum model}

The Lorentzian function for the $i^{t h}$ pool was given by:

$$
L(f)_{i}=\frac{a_{i} b_{i} / \pi}{1+\left(b_{i}\left(f-a_{i}-f_{0}\right)\right)^{2}}
$$


which were fitted to z-spectra according to:

$$
Z(f)=1-\sum_{i=0}^{n-1} c_{i} L(f)_{i}
$$

$a_{i}$ is the estimated frequency offset of the $i^{t h}$ pool, $b_{i}$ is the width parameter, $c_{i}$ is a pool-specific scaling factor and $f_{0}$ is a global frequency offset to account for inhomogeneities in the static magnetic field $\left(B_{0}\right)$. Bayesian maximum likelihood was used to fit $a, b c$ and $f_{0}$ to z-spectra (2), using the fminunc minimization algorithm in Matlab (MathWorks, Massachusetts, US) with the exception of $\mathrm{a}_{0}$ (water pool), which was fixed at 0 ppm. Gaussian prior distributions for each parameter were defined as shown in Table S1, which were derived from Chappell et al. (3).

\section{Tumor volume measurement with MRI}

A $\mathrm{T}_{2}$-weighted, fast spin echo sequence was used for tumor localization and tumor volume measurements, which included the following parameters: repetition time (TR), 1.5s; echo train length, 4; effective echo time ( $\left(T E_{\text {eff }}\right), 17.2 \mathrm{~ms}$; slice thickness, $1 \mathrm{~mm}$; number of slices, 20; number of averages, 4; matrix size, 128x128; field of view (FOV), 30x30 mm². Regions of interest (ROIs) were drawn, covering the entire tumor mass (Matlab). The number of pixels in these volumetric ROIs was multiplied by the voxel volume $(238 \times 238 \times 1000$ $\mu \mathrm{m}^{3}$ ) to estimate tumor volume.

\section{Diffusion MRI acquisition and post-processing}

A multi-slice diffusion-weighted fast spin echo sequence was used which included the following parameters: TR, 1500 ms; TE $E_{\text {eff }}, 2000$ ms; b-value, 150, 300, 503, 760, 1070 s mm$^{-2}$; slice thickness, 1 mm; number of slices, 8; matrix size, 128×128; FOV, $30 \times 30 \mathrm{~mm}^{2}$. The apparent diffusion coefficient (ADC) was quantified from these data by fitting a single exponential to signal intensity values, of the form:

$$
S(b)=S_{0} \exp (-A D C \cdot b)
$$

where $S_{0}$ and $A D C$ are fitted parameters and $b$ is the b-value. This was undertaken on a pixel-by-pixel basis, and pixels were fitted using a maximum likelihood algorithm that took into account the Rician noise distribution (4).

\section{$T_{1}$ measurement}

A Look-Locker segmented inversion recovery sequence was used to estimate the longitudinal relaxation time, $T_{1}$, with the following parameters: number of inversion points, 50; TR, $110 \mathrm{~ms}$; TE, $1.18 \mathrm{~ms}$; slice thickness, $1 \mathrm{~mm}$; number of slices, 8 ; matrix size, 128x128; FOV, $30 \times 30 \mathrm{~mm}^{2}$. In order to sample the longitudinal magnetization during its recovery following an inversion pulse, the Look-Locker sequence applies a train of small flip angle readout pulses, separated by a fixed inversion time (TI). This pulse train partially saturates the signal, resulting in an apparent longitudinal relaxation time, $T_{1}{ }^{*}$, shorter than the true $T_{1}$ time. $T_{1}{ }^{*}$ was estimated by fitting data to the following three parameter model:

$$
S(b)=S_{0}\left(1-\beta \exp \left(-T I / T_{1}^{*}\right)\right)
$$


$T_{1}$ was then estimated according to (5):

$$
T_{1}=T_{1}^{*}(\beta-1)
$$

\section{$T_{2}$ measurement}

A multi-echo multi-slice imaging sequence was used to estimate $T_{2}$, using the following parameters: TR, $1500 \mathrm{~ms} ; 16$ echo times (TE) ranging from 8 to $132 \mathrm{~ms}$, at $8 \mathrm{~ms}$ intervals; slice thickness, $1 \mathrm{~mm}$; number of slices, 8 ; matrix size, $128 \times 128$; FOV, $30 \times 30 \mathrm{~mm}^{2} . T_{2}$ and $S_{0}$ values were estimated using the following equation:

$$
S(T E)=S_{0} \exp \left(-T E / T_{2}\right)
$$

where $S_{0}$ and $T_{2}$ are fitted parameters. Again, a maximum likelihood algorithm was used, which took into account the Rician noise distribution of MRI magnitude data (2).

\section{Immunohistochemistry}

Staining and Imaging. GADD34 and cleaved caspase-3 immunohistochemistry was performed using a Ventana Discovery XT, with the Ventana DAB Map Kit (760-124). Details of the protocols used are provided in supplemental methods. Heat induced epitope retrieval was performed using an EDTA buffer (pH8.0). AntiGADD34 (Santa Cruz Biotech SC-8327) primary antibody incubation was for 4 hours using a 1:50 dilution, followed by amplification with the Ventana Amplification Kit (760-080). Swine anti-Rabbit (Dako E0353) secondary antibody incubation was for 30min, using a 1:200 dilution. Anti-Cleaved-Caspase-3 (Cell Signaling, 9661L) primary antibody incubation was for 30 minutes using a 1:100 dilution. Swine anti-Rabbit (Dako E0353) secondary antibody incubation was for 30 minutes, using a 1:200 dilution. All slides were haematoxylin counterstained and digitalized using LEICA SCN400 scanner (LEICA Microsystems, Milton Keynes, UK), with x40 magnification, 65\% image compression.

Analysis and quantification. Slides were analyzed and quantified using Definines Tissue Studio and Developer (Definines AG, Munich, Germany). Marker expression was quantified by measuring percentage area and percentage nuclei of the positive regions. For the percentage area calculation, distinctive regions of a tumor tissue slide were first manually classified from a sample slide. A typical immunostained tumor tissue was classified into five regions: positive, viable, necrotic, dermis/fat, and glass, in the training process of Tissue Studio composer actions. The software then automatically identified and segmented each slide into five regions of interest (ROI) with threshold based on the intensity of haematoxylin (blue) and chromogen (brown). After the automatic segmentation of all slides was completed, the slides were reassessed manually by the user to correct any mismatched regions (in 'correction mode'). Automatic quantification was then carried out, where the absolute area and percentage area of each ROI over total tissue was calculated, where the total tissue area was the sum of positive, viable and necrotic areas. For the percentage nuclei quantification, in the software training process, tumor tissue area (from segmentation described above) were classified as 'nuclei' or 'cytoplasm', and within the 'nuclei', they were further classified into 'positive', 'viable' and 'necrotic'. The tissues were automatically labeled based on the preset threshold values of the stain intensity. The absolute number and percentage of each class of nuclei were automatically quantified, where the total of nuclei were the sum of positive, viable and necrotic nuclei. The percentage expression of caspase 3 and GADD34 were calculated by taking a mean value between the percentage area and percentage nuclei. 


\section{References}

1. Prinz H. Hill coefficients, dose-response curves and allosteric mechanisms. J Chem Biol. 2010;3(1):37-

44.

2. Walker-Samuel S, Orton M, McPhail LD, Boult JK, Box G, Eccles SA, et al. Bayesian estimation of changes in transverse relaxation rates. Magnetic resonance in medicine. 2010;64(3):914-21.

3. Chappell MA, Donahue MJ, Tee YK, Khrapitchev AA, Sibson NR, Jezzard P, et al. Quantitative Bayesian model-based analysis of amide proton transfer MRI. Magn Reson Med. 2013;70(2):556-67.

4. Walker-Samuel S, Orton M, McPhail LD, Robinson SP. Robust estimation of the apparent diffusion coefficient (ADC) in heterogeneous solid tumors. Magnetic resonance in medicine. 2009;62(2):420-9.

5. Deichmann R, Haase A. Quantification of T1 Values by Snapshot-Flash Nmr Imaging. J Magn Reson. 1992;96(3):608-12.

Table

\begin{tabular}{|c|c|c|c|}
\hline \multirow{2}{*}{ Pool (index) } & \multicolumn{3}{|c|}{ Gaussian prior parameters: mean (s.d.) } \\
\hline & $b(\mathrm{~ms})$ & $a$ (ppm) & $f_{0}(\mathrm{ppm})$ \\
\hline Water (0) & $5(3)$ & $\mathrm{n} / \mathrm{a}$ & \multirow[t]{5}{*}{$0(3)$} \\
\hline Amide (1) & $8(5)$ & $3.5(0.6)$ & \\
\hline Amine (2) & $8(5)$ & $2.4(0.6)$ & \\
\hline Hydroxyl (3) & $5(5)$ & $1.2(0.6)$ & \\
\hline MT \& NOE (4) & $0.1(1)$ & $-2.4(0.6)$ & \\
\hline
\end{tabular}

Table S1. Gaussian prior distribution parameter values for the maximum likelihood model, expressed as mean (s.d.) (derived from Chappell et al. (26)). Entries marked with 'n/a' were fixed (see text for details). 
I. SW1222:
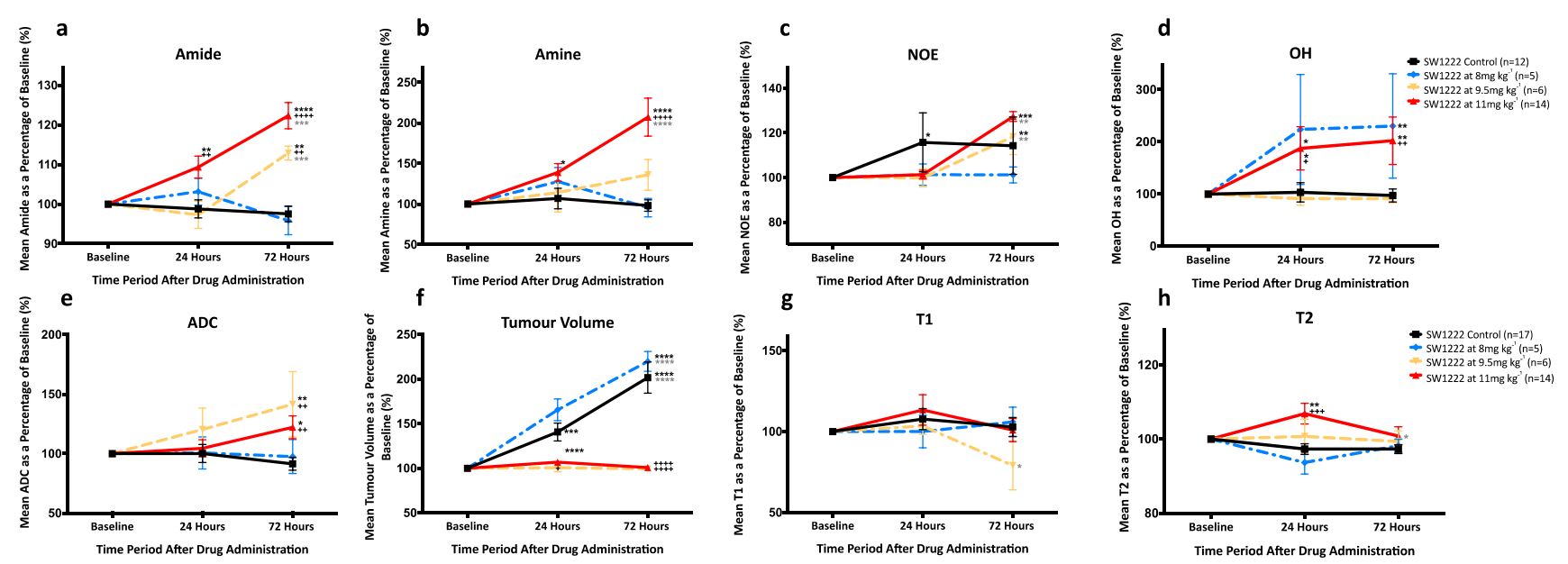

\section{LS174T:}
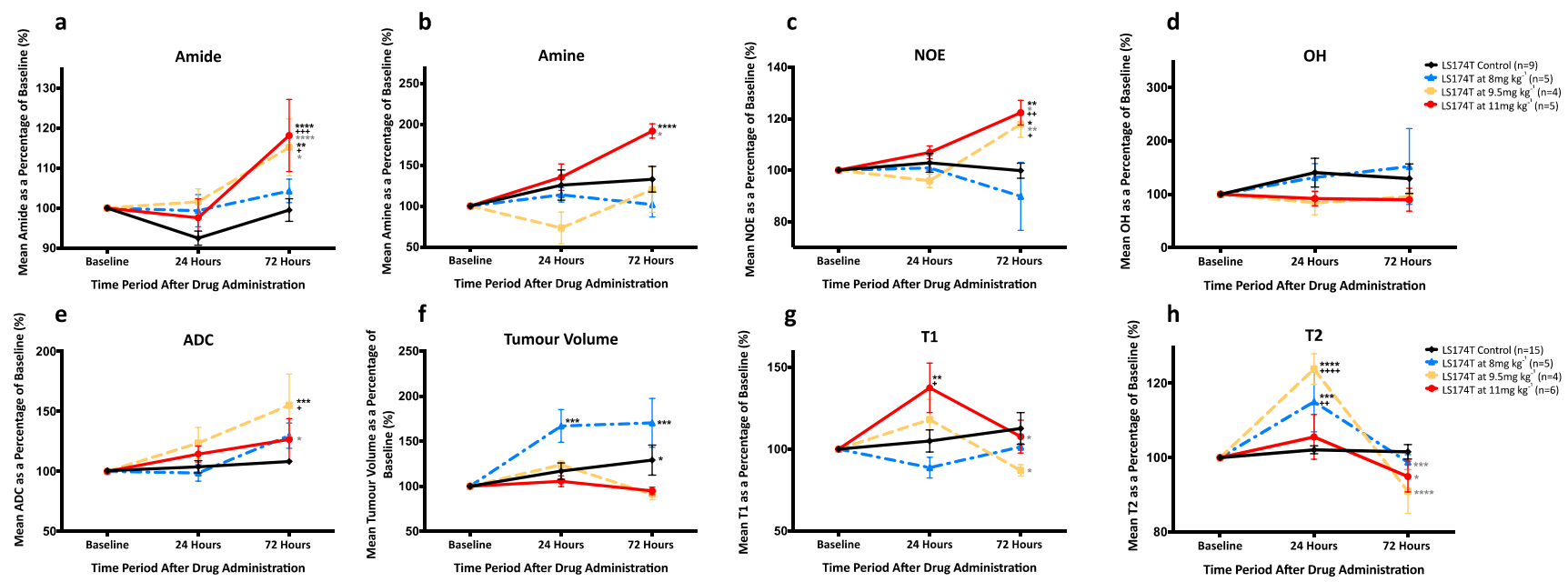

Figure S1. Graphs showing the relationship between the relative change in each non-invasive imaging parameter and time, for three different doses of Ixazomib (8, 9.5 and $\left.11 \mathrm{mg} \mathrm{kg}^{-1}\right)$ and vehicle, in (I) SW1222 and (II) LS174T tumours. Imaging parameters shown are: (a) median amide area, (b) median amine area, (c) median NOE area, (d) median $\mathrm{OH}$ area, (e) median ADC, (f) median volume, (g) median T1 and (h) median T2. Error bars correspond to the standard error of the mean $\left({ }^{*} \mathrm{P}<0.05, * * * \mathrm{P}<0.01, * * * * \mathrm{P}<0.001 ; *(\right.$ black $)=$ compared to baseline, ${ }^{*}$ (grey) $=$ compared 24 hours after ixazomib treatment, ${ }^{+}=$compared to control). 